\title{
Angle between the common and internal carotid arteries detected by ultrasound is related to intima-media thickness among those with atherosclerotic disease
}

\author{
Satoshi Daitoku $\cdot$ Toshinori Yuasa $\cdot$ Hiroshi Tsunenari $\cdot$ Shigeho Maenohara \\ Kazuharu Mine • Yuichi Tamatsu • Kazuyuki Shimada • \\ Chihaya Koriyama $\cdot$ Mitsuru Ohishi $\cdot$ Masahisa Horiuchi
}

Received: 3 December 2014 / Accepted: 19 February 2015/Published online: 13 March 2015

(C) The Japanese Society for Hygiene 2015

\begin{abstract}
Objectives Although carotid artery structural variations have been detected by ultrasound, their clinical significance is not fully understood. The objective of this study was to determine whether the angle between the common carotid artery (CCA) and the internal carotid artery (ICA), designated angle $\alpha$, an ultrasound-detectable carotid artery structural variation, is related to carotid artery intima-media thickness (IMT), a surrogate marker for carotid atherosclerosis.
\end{abstract}

Electronic supplementary material The online version of this article (doi:10.1007/s12199-015-0453-7) contains supplementary material, which is available to authorized users.

S. Daitoku · M. Horiuchi $(\square)$

Department of Hygiene and Health Promotion Medicine, Kagoshima University Graduate School of Medical and Dental Sciences, 8-35-1 Sakuragaoka, Kagoshima 890-8544, Japan e-mail: masakun@m.kufm.kagoshima-u.ac.jp

S. Daitoku $\cdot$ T. Yuasa $\cdot$ M. Ohishi $(\bowtie)$

Department of Cardiovascular Medicine and Hypertension, Kagoshima University Graduate School of Medical and Dental Sciences, 8-35-1 Sakuragaoka, Kagoshima 890-8544, Japan e-mail: ohishi@m2.kufm.kagoshima-u.ac.jp

S. Daitoku $\cdot$ H. Tsunenari $\cdot$ S. Maenohara

Kagoshima Kouseiren Hospital, Kagoshima, Japan

K. Mine $\cdot$ Y. Tamatsu $\cdot$ K. Shimada

Department of Gross Anatomy Section,

Kagoshima University Graduate School of Medical

and Dental Sciences, Kagoshima, Japan

C. Koriyama

Department of Epidemiology and Preventive Medicine,

Kagoshima University Graduate School of Medical and Dental

Sciences, Kagoshima, Japan
Methods As a cross-sectional study, we measured angle $\alpha$ in routine carotid artery ultrasounds from 176 subjects (130 men) with atherosclerotic disease/risk factors that attended Kouseiren Hospital in Kagoshima City, Japan between August 2007 and April 2009. We evaluated the correlation between the angle $\alpha$ and CCA- or ICA-IMT.

Results Angle $\alpha$ was weakly correlated with age but significantly correlated with ICA-IMT. The correlation was stronger in subjects with an ICA-IMT $\geq 0.5 \mathrm{~mm}$ than in those with an ICA-IMT $<0.5 \mathrm{~mm}$ (Right side $r=0.475$ vs. 0.246 , Left side $r=0.498$ vs. 0.301 , respectively). Upon multivariate logistic regression analysis, angle $\alpha$ and serum low-density lipoprotein cholesterol were independent explanatory variables for ICA-IMT.

Conclusion Angle $\alpha$ is related to ICA-IMT in subjects with atherosclerotic disease or risk factors in this study.

Keywords Atherosclerotic risk - Cross-sectional study Intima-media thickness · Vessel structural variations

\section{Introduction}

Intima-media thickness (IMT) is an important surrogate marker for atherosclerosis-based stroke and cardiovascular disease [1, 2]. Many factors can increase IMT, including conventional vascular risk factors such as hypercholesterolemia and hypertension [3, 4]. However, increased IMT cannot be explained by conventional vascular risk factors alone. Limited evidence suggests that local hemodynamic factors associated with the structural variations are involved in atheroma pathogenesis [5-7]. Thus, vessel structural variations should be regarded as risk factors for increased IMT [8]. In fact, Sitzer et al. [9] reported that angle variations of internal carotid artery (ICA) origin, 
detected by ultrasound, may account for some of the unexplained predisposition towards increased IMT and plaque formation in healthy subjects. However, the clinical significance of these structural variations has not been fully elucidated.

Diagnostic imaging, including magnetic resonance imaging (MRI), computed tomography (CT), angiography, and ultrasound, has shown that carotid vessels exhibit a variety of anatomic configurations [10-12]. Although structural variations have been identified by MRI, CT, and angiography, carotid artery ultrasound detection may be more clinically useful because it is portable and relatively inexpensive $[13,14]$. In the present study, we measured the angle between the common carotid artery (CCA) and the ICA in Japanese subjects with atherosclerotic risk factors and/or atherosclerotic disease. We determined whether this angle is related to carotid artery IMT, a surrogate marker for carotid atherosclerosis.

\section{Materials and methods}

\section{Study subjects}

We retrieved data for 176 subjects (130 men) who underwent successful routine carotid artery ultrasound examinations and blood tests at Kouseiren Hospital in Kagoshima City, Japan from August 2007 to April 2009. A total of 206 subjects underwent carotid artery ultrasound during this time period; however, 30 subjects lacked blood tests because they were less than 35 years. These subjects were excluded from the study. We analyzed the previously collected data including the ultrasound and blood tests. This study, including the waiver of informed consent, was approved by our institutional review board, the Ethics Committee for Clinical Examination at Kouseiren Hospital.

The subjects' clinical status and lifestyle were classified as follows. Each subject was classified as a current smoker, former smoker, or never smoker based on self-reporting. Subjects with hypertension were defined as those with $>140 / 90 \mathrm{mmHg}$ blood pressure on the day of the ultrasound examinations or those receiving antihypertensive medications. Subjects with dyslipidemia were defined as those receiving antidyslipidemic medications, including statins, or those with the following serum values: lowdensity lipoprotein cholesterol (LDL-C) $>140 \mathrm{mg} / \mathrm{dL}$, high-density lipoprotein cholesterol $<40 \mathrm{mg} / \mathrm{dL}$, or triglycerides $>150 \mathrm{mg} / \mathrm{dL}$. LDL-C was calculated using the Friedewald equation in patients with triglycerides $<400 \mathrm{mg} / \mathrm{dL}$ [15]. Subjects with diabetes mellitus were defined as those receiving antidiabetic medications or those with a fasting blood glucose $>126 \mathrm{mg} / \mathrm{dL}$ or an NGSPstandardized glycated hemoglobin $(\mathrm{HbA} 1 \mathrm{c})>6.5 \%$.
Carotid artery ultrasound

The standard two-dimensional and Doppler ultrasound was routinely performed using a commercially available machine (SSA-700A Aplio 50; Toshiba Co. Ltd., Tokyo, Japan) with a linear $(8.5 \mathrm{MHz})$ or micro-convex $(5.0 \mathrm{MHz})$ probe [16]. The sonography specialist visualized the region from the proximal CCA to the distal ICA into the carotid duct. We measured the angle between the CCA and ICA in 176 subjects from the previously collected data. Specifically, we measured the angle according to the center lines of the CCA and ICA using an image in plane with the two arteries, obtained during the examination (Fig. 1a). This angle was designated angle $\alpha$. We measured the IMT at the near wall of the proximal ICA (Fig. 1a) and at the middle position of the CCA, meaning the CCA position $2.0 \mathrm{~cm}$ proximal to the bifurcation (Fig. $1 \mathrm{~b}$ ). These two IMT determinations were designated the ICA-IMT and the CCA-IMT, respectively. The CCA inner diameter, another potential vessel structural variation, was measured $2.0 \mathrm{~cm}$ proximal to the bifurcation during the contraction phase using tomography (Fig. 1b) [17]. A blinded, Japanese government-qualified sonographer evaluated the carotid artery ultrasound images.

Statistical analysis

Values are shown as mean \pm standard deviation. A twosided paired or unpaired Student's $t$ test was used, as appropriate, to compare the values between groups, and the Pearson's product-moment correlation coefficient ( $r$ value) was used to evaluate the correlation between values. A Chi-square test was performed on the categorical variables. For the smoking status, analysis was performed using one-way analysis of variance (ANOVA). Multivariate logistic regression analyses were conducted to evaluate age, BMI, angle $\alpha$, LDL-C, smoking status, hypertension, and diabetes as explanatory variables for ICA-IMT. StatMate III (ATMS Co., Tokyo, Japan) or Stata 8.1 (Stata Co., College Station, TX) was used, as appropriate, for the statistical analyses. $P<0.05$ was considered statistically significant.

\section{Results}

Subject characteristics

Table 1 presents the characteristics of the subjects (130 men and 46 women) enrolled in the present study. The mean age was $61.8 \pm 10.3$ years. All subjects were $\geq 35$ years old. Based on the clinical history, all subjects had atherosclerotic risk factors and/or atherosclerotic 
Fig. 1 Measurement of a angle $\alpha$, the angle between the internal carotid artery (ICA) and common carotid artery (CCA), and $\mathbf{b}$ intima-media thickness (IMT). In (a), dotted lines indicate the vessels' center lines. Angle $\alpha$ is designated to be the angle formed by the intersection of these two lines. Representative cases of a relatively small angle (left) and large angle (right) are shown. IMT at the proximal ICA near wall is marked ICA-IMT. In (b), the location where CCA-IMT and CCA diameter are measured is shown with a white square. The dotted line with double arrowheads indicates the location $2.0 \mathrm{~cm}$ proximal from the vessel bifurcation. The solid line with double arrowheads and double dotted lines in the enlarged view (right) indicate the CCA diameter and CCAIMT, respectively
A Small angle

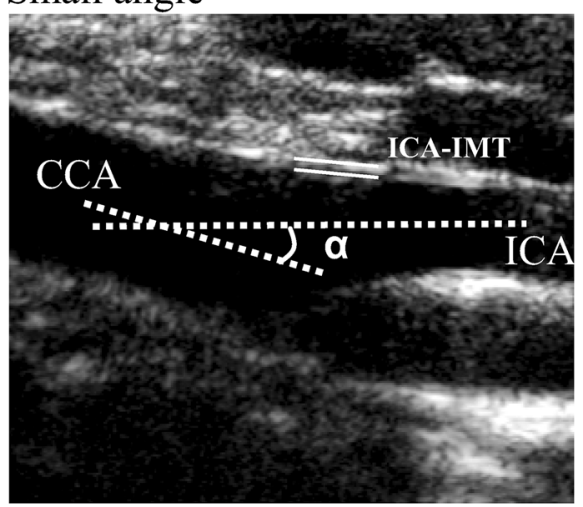

Large angle

B

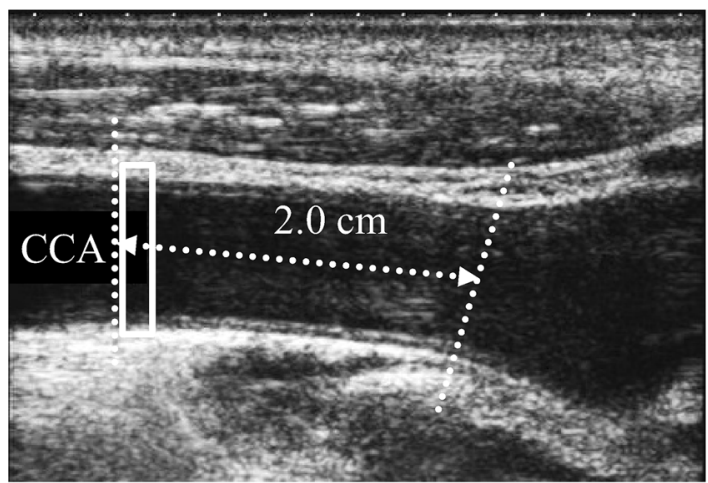

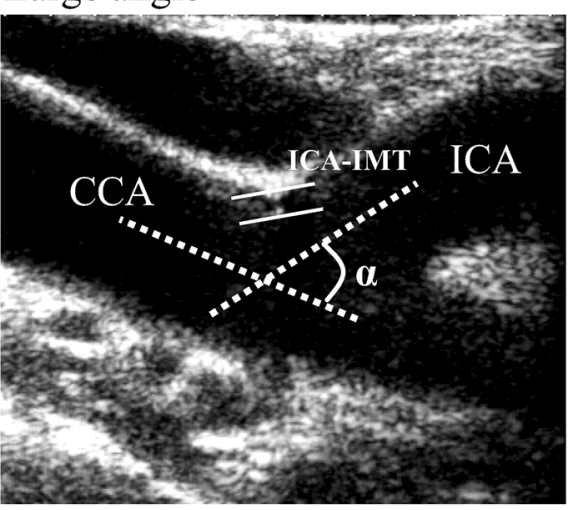

Enlarged

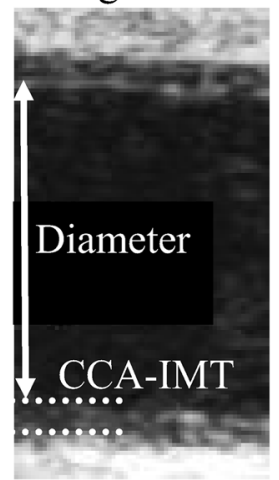

Table 1 Baseline characteristics of the 176 subjects

\begin{tabular}{ll}
\hline Age (years) & $61.8 \pm 10.3$ \\
Men/women & $130(73.9) / 46(26.1)$ \\
BMI (kg/m2) & $23.7 \pm 3.4$ \\
BSA (m2) & $1.6 \pm 0.2$ \\
Systolic BP (mm Hg) & $135 \pm 19$ \\
Diastolic BP (mm Hg) & $80 \pm 14$ \\
Smoking & \\
Never & $86(48.9)$ \\
Former & $43(24.4)$ \\
Current & $47(26.7)$ \\
Hypertension & $157(89.2)$ \\
Dyslipidemia & $88(50.0)$ \\
Diabetes mellitus & $117(66.4)$ \\
CAD & $62(35.2)$ \\
PAD & $50(28.4)$ \\
Stroke & $36(20.5)$ \\
\hline
\end{tabular}

Values are shown as mean \pm standard deviation or number $(\%)$ $B M I$ body mass index, $B P$ blood pressure, $B S A$ body surface area, $C A D$ coronary artery disease, $P A D$ peripheral artery disease. Smoking condition numbers based on self-reported data and are defined as follows: Never never smoked a cigarette, Former previously smoked, Current smoking at the time of the study diseases (Table 1). Approximately three-quarters of the subjects were never smokers or former smokers.

Assessment of carotid artery structural variations

We measured the angle between the CCA and ICA (Fig. 1) in 176 subjects. The results showed significant laterality. Angle $\alpha$ of the right carotid artery was smaller than the left [right $22.3 \pm 12.7$ (range 5.1-70.0) degrees vs. left $26.3 \pm 14.7$ (1.6-79.1) degrees, $P<0.01$ using a paired Student's $t$ test]. However, the CCA inner diameter did not differ significantly between the right and left carotid artery (right $3.83 \pm 0.61 \mathrm{~mm} / \mathrm{m}^{2}$ vs. left $3.77 \pm 0.56 \mathrm{~mm} / \mathrm{m}^{2}$, $P=0.27$ using a paired Student's $t$ test). Angle $\alpha$ was significantly correlated with age in both the right and left carotid artery (Fig. 2). However, in both the right and left carotid arteries, the correlation between age and CCA diameter/body surface area (BSA)was greater than the correlation between age and angle $\alpha$. As shown in Table 2, the ICA-IMT around the near wall of both the right and left ICA significantly correlated with angle $\alpha$. Furthermore, angle $\alpha$ was significantly correlated with the CCA diameter on both sides (right $r=0.244, P<0.01$; left $r=0.233$, 
Fig. 2 Correlation between angle $\alpha$ and age (a, b) and CCA diameter/body surface area (BSA) and age (C, D). Right and left represent the vessel position. Open circles represent actual values. Values for $r$ (Pearson's product-moment correlation coefficient) and $P$ are shown
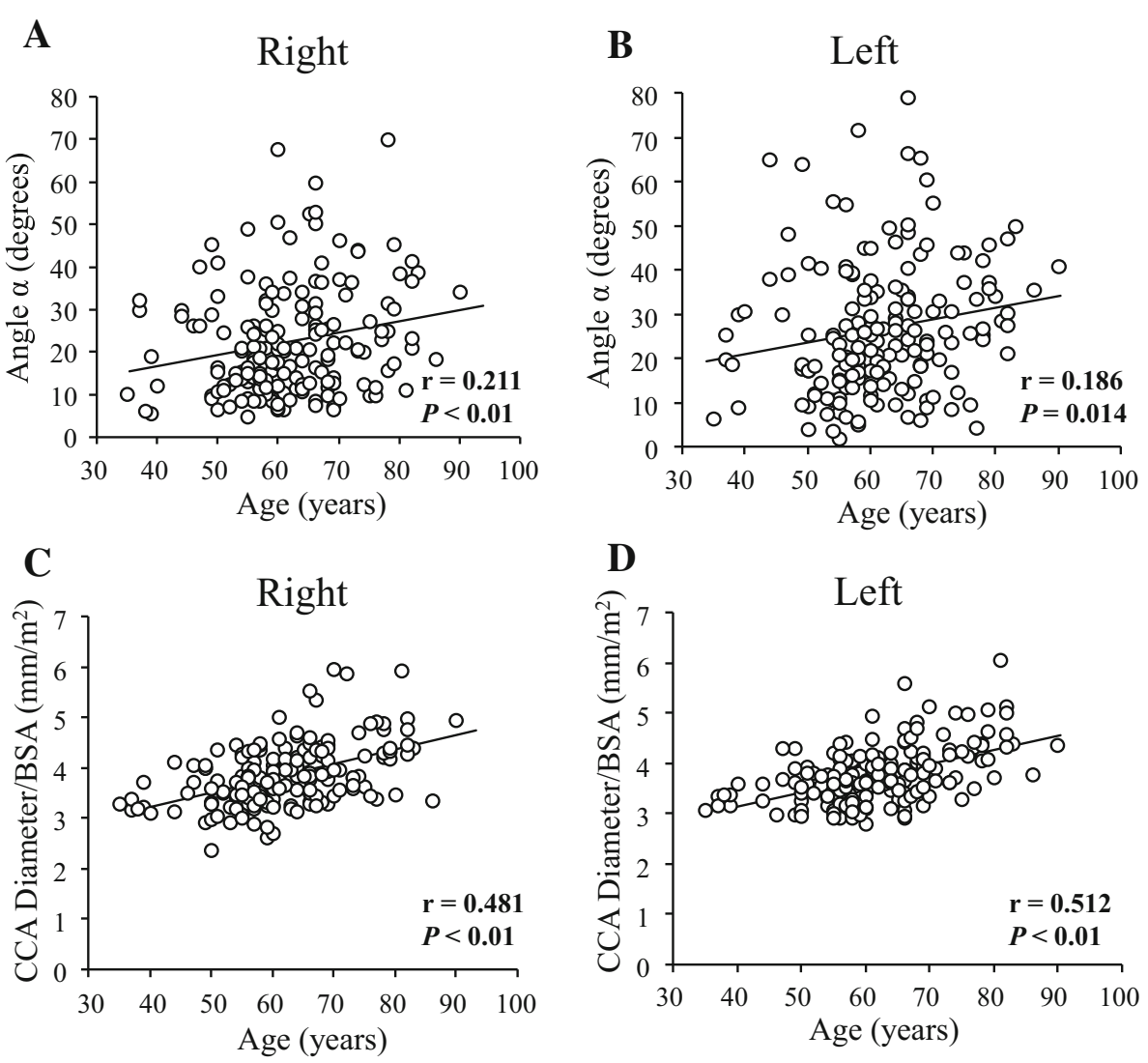

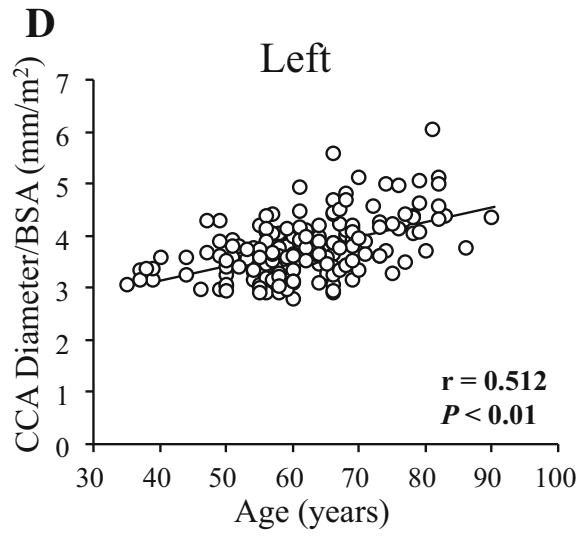

Table 2 Correlations between angle $\alpha$ and CCA-IMT or ICA-IMT in the right and left carotid arteries

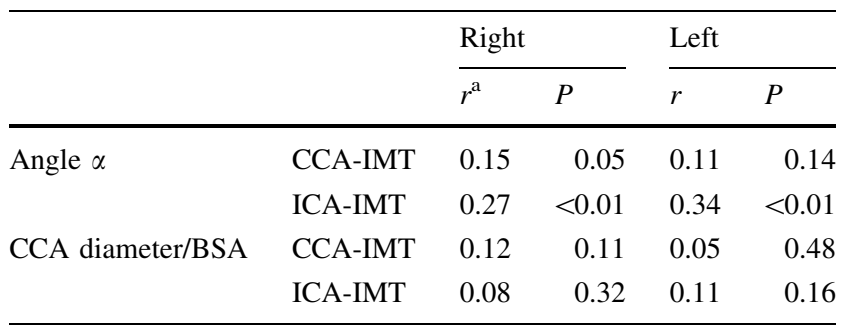

${ }^{a} r$ denotes the Pearson correlation coefficient $(N=176)$

$C C A$ common carotid artery, ICA intra carotid artery, BSA body surface area

$P<0.01)$. There were no significant angle $\alpha$ differences in subjects with or without hypertension, dyslipidemia, diabetes mellitus, coronary artery disease, peripheral artery disease, or stroke (Supplementary Table 1). On the right, the CCA diameter/BSA values according to smoking status were not significant: never $3.9 \pm 0.5$; former $3.8 \pm 0.8$; and current $3.8 \pm 0.6 \mathrm{~mm} / \mathrm{m}^{2}$ (one-way ANOVA). Similarly, on the left, the CCA diameter/BSA values were not significant: never, $3.8 \pm 0.5$; former, $3.8 \pm 0.6$; and current, $3.8 \pm 0.6 \mathrm{~mm} / \mathrm{m}^{2}$ (one-way ANOVA).

The correlation between angle $\alpha$ and ICA-IMT clearly indicated the existence of the two groups, with a differentiating value of $0.5 \mathrm{~mm}$ ICA-IMT (Fig. 3). This number is similar to the previously reported mean value in obese Japanese subjects [18]. Therefore, we recalculated the correlation values with the subjects classified into two groups: ICA-IMT $<0.5 \mathrm{~mm}$ and ICA-IMT $\geq 0.5 \mathrm{~mm}$. The correlation between angle $\alpha$ and ICA-IMT was stronger for the ICA-IMT $\geq 0.5 \mathrm{~mm}$ group. Furthermore, ICAIMT $\geq 0.5 \mathrm{~mm}$ subjects had significantly higher total cholesterol and LDL-C values than those in the ICAIMT $<0.5 \mathrm{~mm}$ group (Table 3 ). Moreover, multivariate logistic regression analysis including age, BMI, angle $\alpha$, LDL-C, smoking status, hypertension, and diabetes revealed that angle $\alpha$ and serum LDL-C were independent explanatory variables for ICA-IMT (Table 4).

\section{Discussion}

In the present study, we used carotid artery ultrasound to measure the angle between the CCA and ICA, angle $\alpha$, in both the right and left carotid arteries. The angle was significantly correlated with ICA-IMT, a surrogate marker for carotid atherosclerosis. This result was consistent with a report on healthy subjects by Sitzer et al. [9]. Moreover, we found that the correlation between angle $\alpha$ and ICA-IMT was stronger in subjects with an IMT $\geq 0.5 \mathrm{~mm}$ than in those with an ICA-IMT $<0.5 \mathrm{~mm}$. Multivariate logistic 
Fig. 3 Correlation between angle $\alpha$ and ICA-IMT $(\mathbf{a}, \mathbf{b})$ and CCA diameter/BSA and ICAIMT (c, d). Right and left represent the vessel position. Open and closed circles represent values from the ICAIMT $<0.5 \mathrm{~mm}$ group and ICAIMT $\geq 0.5 \mathrm{~mm}$ group, respectively. Solid, shortdashed, and long-dashed lines denote the correlation line for the total subjects, the ICAIMT $<0.5 \mathrm{~mm}$ group subjects, and the ICA-IMT $\geq 0.5 \mathrm{~mm}$ group subjects, respectively. For (A, B), the $r$ and $P$ values for each group are shown to the right of each correlation line. For (c, d), the $r$ and $P$ values for the ICA-IMT $<0.5 \mathrm{~mm}$ group and ICA-IMT $\geq 0.5 \mathrm{~mm}$ group are shown to the right of the correlation line and total subjects are shown to the left
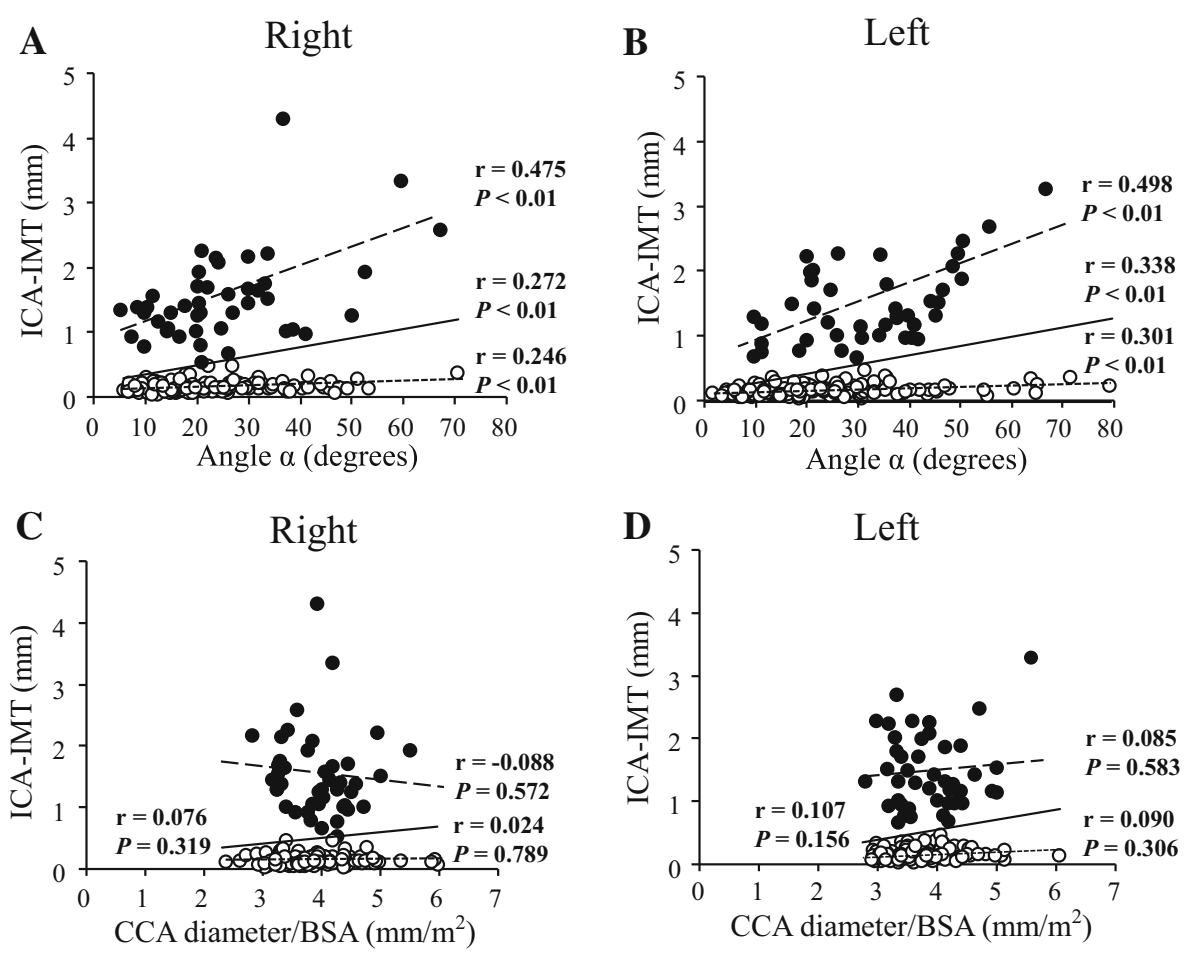

Table 3 Differences in angle $\alpha$, patient characteristics, and blood test results between the ICA-IMT $<0.5 \mathrm{~mm}$ and ICAIMT $\geq 0.5 \mathrm{~mm}$ groups
Values are shown as mean \pm standard deviation or number (\%). Statistical analyses included unpaired Student's $t$ tests (two-sided) or Chi-square tests for categorical variables. $(N=176)$

$T G$ triglyceride, $T C$ total cholesterol, $L D L-C$ low-density lipoprotein cholesterol, $H D L-C$ high-density lipoprotein cholesterol

\begin{tabular}{|c|c|c|c|c|c|c|}
\hline & \multicolumn{3}{|l|}{ Right } & \multicolumn{3}{|l|}{ Left } \\
\hline & $<0.5 \mathrm{~mm}$ & $\geq 0.5 \mathrm{~mm}$ & $P$ & $<0.5 \mathrm{~mm}$ & $\geq 0.5 \mathrm{~mm}$ & $P$ \\
\hline Angle $\alpha$ (degrees) & $21.2 \pm 12.2$ & $25.5 \pm 13.8$ & 0.05 & $24.3 \pm 14.6$ & $33.2 \pm 13.7$ & $<0.01$ \\
\hline Age (years) & $61.5 \pm 10.1$ & $62.5 \pm 11.1$ & 0.57 & $61.7 \pm 10.2$ & $62.0 \pm 10.6$ & 0.88 \\
\hline Men/women & $97 / 35$ & $33 / 11$ & 0.84 & $98 / 34$ & $32 / 12$ & 0.84 \\
\hline Smoking & & & 0.06 & & & 0.06 \\
\hline Never & $59(44.7)$ & $27(62.4)$ & & $59(44.7)$ & $31(70.5)$ & \\
\hline Former & $35(26.5)$ & $8(18.2)$ & & $35(26.5)$ & $9(20.5)$ & \\
\hline Current & $38(28.8)$ & $9(20.5)$ & & $38(28.8)$ & $9(20.5)$ & \\
\hline Hypertension & $120(90.9)$ & $37(84.1)$ & 0.20 & $121(91.7)$ & $36(81.8)$ & 0.07 \\
\hline Dyslipidemia & $75(56.8)$ & $13(29.5)$ & $<0.01$ & $76(57.6)$ & $12(27.3)$ & $<0.01$ \\
\hline Diabetes mellitus & $91(68.9)$ & $26(59.1)$ & 0.23 & $92(70.0)$ & $25(56.8)$ & 0.11 \\
\hline CAD & $49(37.1)$ & $13(29.5)$ & 0.36 & $48(36.4)$ & $14(31.8)$ & 0.58 \\
\hline PAD & $38(28.8)$ & $12(27.2)$ & 0.84 & $39(27.8)$ & $11(25)$ & 0.56 \\
\hline Stroke & $30(22.7)$ & $6(13.6)$ & 0.20 & $30(22.7)$ & $6(13.6)$ & 0.20 \\
\hline $\mathrm{TG}(\mathrm{mg} / \mathrm{dL})$ & $130.3 \pm 83.5$ & $120.8 \pm 66.0$ & 0.49 & $128.8 \pm 81.7$ & $125.2 \pm 73.0$ & 0.79 \\
\hline $\mathrm{TC}(\mathrm{mg} / \mathrm{dL})$ & $196.3 \pm 36.6$ & $211.1 \pm 39.1$ & 0.02 & $195.8 \pm 36.9$ & $212.8 \pm 37.4$ & $<0.01$ \\
\hline LDL-C (mg/dL) & $113.5 \pm 33.5$ & $130.8 \pm 31.3$ & $<0.01$ & $113.2 \pm 33.6$ & $131.5 \pm 30.3$ & $<0.01$ \\
\hline HDL-C (mg/dL) & $56.8 \pm 15.0$ & $56.2 \pm 16.1$ & 0.82 & $56.8 \pm 15.0$ & $56.3 \pm 16.0$ & 0.84 \\
\hline
\end{tabular}

regression analysis revealed that, in addition to serum LDL-C, angle $\alpha$ was an independent explanatory variable for ICA-IMT.

The angle $\alpha$ structural variation has been examined previously with respect to its clinical significance. In a study by Sitzer et al. [9], it was referred to as the angle of ICA origin and correlated with ICA-IMT in healthy subjects. However, in that study, the angle was measured using a cross-sectional-axis view from the carotid artery ultrasound. In this study, we used a longitudinal-axis view to measure the angle from previously collected data. The longitudinal-axis view is easier than the cross-sectionalaxis view, and the value could be measured from the previously collected data; therefore, we selected longitudinal- 
Table 4 Results of multivariate logistic regression analysis of ICA-IMT for different values of angle $\alpha$ or LDL-cholesterol

\begin{tabular}{|c|c|c|c|c|c|c|c|c|c|c|c|}
\hline \multicolumn{6}{|c|}{ Right ICA-IMT } & \multicolumn{6}{|c|}{ Left ICA-IMT } \\
\hline Group & $N$ & Angle $\alpha$ (degrees) & Odds ratio & CI & $P$ & Group & $N$ & Angle $\alpha$ (degrees) & Odds Ratio & CI & $P$ \\
\hline \multicolumn{6}{|c|}{$P$ for trend $=0.050$} & \multicolumn{6}{|c|}{$P$ for trend $<0.010$} \\
\hline 0 & 60 & $10.5 \pm 2.5$ & 1 (reference) & & & 0 & 59 & $12.0 \pm 4.5$ & 1 (reference) & & \\
\hline 1 & 59 & $19.8 \pm 3.4$ & 2.45 & $0.93-6.43$ & 0.069 & 1 & 59 & $24.0 \pm 3.4$ & 1.64 & $0.56-4.81$ & 0.368 \\
\hline 2 & 57 & $37.3 \pm 10.2$ & 2.73 & $1.01-7.37$ & 0.047 & 2 & 58 & $43.2 \pm 11.3$ & 6.07 & $2.11-17.41$ & $<0.010$ \\
\hline Group & $N$ & LDL-C (mg/dL) & Odds ratio & $\mathrm{CI}$ & $P$ & Group & $N$ & LDL-C (mg/dL) & Odds Ratio & CI & $P$ \\
\hline \multicolumn{6}{|c|}{$P$ for trend $<0.010$} & \multicolumn{6}{|c|}{$P$ for trend $=0.012$} \\
\hline 0 & 59 & $80.5 \pm 17.2$ & 1 (reference) & & & 0 & 59 & $80.5 \pm 17.2$ & 1 (reference) & & \\
\hline 1 & 59 & $118.4 \pm 7.3$ & 4.24 & $1.43-12.56$ & $<0.010$ & 1 & 59 & $118.4 \pm 7.3$ & 4.30 & $1.41-13.15$ & 0.010 \\
\hline 2 & 58 & $155.1 \pm 16.5$ & 5.17 & $1.74-15.41$ & $<0.010$ & 2 & 58 & $155.1 \pm 16.5$ & 4.44 & $1.46-13.48$ & $<0.010$ \\
\hline
\end{tabular}

Values are shown as mean \pm standard deviation. Multivariate logistic regression analyses including age, BMI, angle $\alpha$, LDL-C, smoking status, hypertension, and diabetes as explanatory variables were performed. $N=176$ subjects. The subjects were divided into three groups containing one-third of the subjects each: Group 0, (the reference group), Group 1, and Group 2, based on increasing angle $\alpha$ or LDL-C values. Odds ratios were calculated by defining subjects with an ICA-IMT $<0.5 \mathrm{~mm}$ as 0 and subjects with an ICA-IMT $\geq 0.5 \mathrm{~mm}$ as 1 . The trend of association was assessed using a logistic regression model assigning consecutive integers to the angle $\alpha$ or LDL-C values. Additionally, hypertension in left ICA-IMT showed a significant odds ratio value (odds ratio $0.27, \mathrm{CI} 0.08-0.87, P=0.028$ )

$L D L-C$ low-density lipoprotein cholesterol, $C I 95 \%$ confidence interval

axis view measurements for this study. Furthermore, because of the advantage, the longitudinal-axis view may be preferred when performing clinical examinations on a large number of subjects.

The correlation between angle $\alpha$ and age was weaker than the correlation between CCA diameter, another vessel structural variation, and age (Fig. 2). Carotid vessel structural variations are determined by both congenital and lifestyle-related acquired factors [19-24]. In a twin study, vessel configuration was more similar between monozygotic than dizygotic twins [25], indicating that vessel structural variation may be influenced by congenital factors. However, smoking, an acquired factor, can induce vessel remodeling, and therefore, may increase CCA diameter [24]. As angle $\alpha$ showed a significant but weak correlation with age, angle $\alpha$ structural variations may depend more on congenital factors than acquired ones.

Angle $\alpha$ exhibited a greater correlation with ICA-IMT than CCA-IMT, suggesting that angle $\alpha$ 's influence is more pronounced downstream of the blood flow. Vessel structural variations can affect blood flow hemodynamics in a focal manner $[5,6]$. Angle $\alpha$ may induce downstream turbulent flow, depending on the size of the angle. Theoretically, a greater angle may increase the risk of increased IMT due to slower and possibly turbulent blood flow. Although further experiments measuring actual blood flow rates are needed to confirm this theory, the results of this study may support it (Table 2; Fig. 3).

Our observation that there was a stronger correlation between angle $\alpha$ and ICA-IMT in ICA-IMT $\geq 0.5 \mathrm{~mm}$ subjects suggests that IMT size may be influenced by other factors such as risk factors for atherosclerosis (Fig. 3). We did not observe this relationship with the CCA diameter. When we examined clinical and serum biochemistry differences between the subjects with those with $<0.5 \mathrm{~mm}$ ICA-IMT and those with $\geq 0.5 \mathrm{~mm}$ ICA-IMT, subjects with $\geq 0.5 \mathrm{~mm}$ ICA-IMT had higher total cholesterol and LDL-C (Table 3). Additionally, both LDL-C and angle $\alpha$ were independent explanatory factors based on multivariate logistic regression analyses. This suggests that abnormal cholesterol metabolism may be related to IMT, regardless of angle $\alpha$ 's size. Interestingly, the percentage of subjects with dyslipidemia was higher in those with an ICAIMT $<0.5 \mathrm{~mm}$ than in those with an ICA-IMT $\geq 0.5 \mathrm{~mm}$. This finding suggests that antidyslipidemic drugs were effective in lowering total cholesterol and LDL-C [26], as well as limiting IMT. Abnormal cholesterol metabolism is a conventional risk factor for increased IMT in many observational studies [27-29]. In addition, it should be noted that the regression analysis also revealed that hypertension combined with increased left ICA-IMT produced a significant odds ratio value (odds ratio $0.27, P=0.03$ ). However, the odds ratio is lower than 1.0, and the reason for this result is not clear at present.

The present study had two main limitations. First, although angle $\alpha$ is shown to be an IMT-related factor, our study was cross-sectional. The correlations we noted should be examined further in a longitudinal and observational study over a prolonged period. An additional case-control study involving, for example, individuals with a brain 
infarction may also be appropriate. Second, the angle $\alpha$ correlations were not confirmed for atherosclerosis surrogate markers other than ICA-IMT. Accordingly, future studies examining the correlation between angle $\alpha$ and other variables, such as pulse wave velocity (a measure of arterial wall stiffness), when investigating the vessel properties involved in atherosclerosis would be valuable [30, 31].

In conclusion, angle $\alpha$ was effectively measured using longitudinal-axis view carotid artery ultrasound from the data collected. We found that the vessel structure variation, angle $\alpha$, is related to ICA-IMT in Japanese subjects with atherosclerotic risk factors or atherosclerotic disease, especially those with higher serum cholesterol levels.

Acknowledgments We would like to thank the late Prof. Toru Takeuchi for encouraging us to continue with this work and Mr. Daniel Mrozek and Ms. Rinko Kawakami for copyediting. This research was supported by a research fund from Kagoshima University.

Conflict of interest The authors declare that they have no conflict of interest.

\section{References}

1. O'Leary DH, Polak JF, Kronmal RA, Manolio TA, Burke GL, Wolfson SK. Carotid-artery intima and media thickness as a risk factor for myocardial infarction and stroke in older adults. N Engl J Med. 1999;340(1):14-22.

2. Lorenz MW, Markus HS, Bots ML, Rosvall M, Sitzer M. Prediction of clinical cardiovascular events with carotid intima-media thickness: a systematic review and meta-analysis. Circulation. 2007;115(4):459-67.

3. Simons PCG, Algra A, Bots ML, Grobbee DE, van der Graaf Y, The SMART study group. Common carotid intima-media thickness and arterial stiffness: indicators of cardiovascular risk in high-risk patients the SMART study (Second Manifestations of ARTerial Disease). Circulation. 1999;100(9):951-7.

4. Salonen RM, Nyyssönen K, Kaikkonen J, Porkkala-Sarataho E, Voutilainen S, Rissanen TH, et al. Six-year effect of combined vitamin $\mathrm{C}$ and $\mathrm{E}$ supplementation on atherosclerotic progression: the Antioxidant Supplementation in Atherosclerosis Prevention (ASAP) study. Circulation. 2003;107(7):947-53.

5. Glagov S, Zarins C, Giddens DP, Ku DN. Hemodynamics and atherosclerosis. Insights and perspectives gained from studies of human arteries. Arch Pathol Lab Med. 1988;112(10):1018-31.

6. Perktold K, Resch M. Numerical flow studies in human carotid artery bifurcations: basic discussion of the geometric factor in atherogenesis. J Biomed Eng. 1990;12(2):111-23.

7. Schulz UGR, Rothwell PM. Major variation in carotid bifurcation anatomy: a possible risk factor for plaque development? Stroke. 2001;32(11):2522-9.

8. Weibel J, Fields WS. Tortuosity, coiling, and kinking of the internal carotid artery. II. Relationship of morphological variation to cerebrovascular insufficiency. Neurology. 1965;15:462-8.

9. Sitzer M, Puac D, Buehler A, Steckel DA, von Kegler S, Markus HS, et al. Internal carotid artery angle of origin: a novel risk factor for early carotid atherosclerosis. Stroke. 2003;34(4):950-5.

10. Anderson GB, Ashforth R, Steinke DE, Ferdinandy R, Findlay JM. CT angiography for the detection and characterization of carotid artery bifurcation disease. Stroke. 2000;31(9):2168-74.
11. Krishnaswamy A, Klein JP, Kapadia SR. Clinical cerebrovascular anatomy. Catheter Cardiovasc Interv. 2010;75(4):530-9.

12. Aristokleous N, Seimenis I, Papaharilaou Y, Georgiou GC, Brott $\mathrm{BC}$, Eracleous E, et al. Effect of posture change on the geometric features of the healthy carotid bifurcation. IEEE Trans Inf Technol Biomed. 2011;15(1):148-54.

13. Lehmann ED, Hopkins KD, Rawesh A, Joseph RC, Kongola K, Coppack SW, et al. Relation between number of cardiovascular risk factors/events and noninvasive Doppler ultrasound assessments of aortic compliance. Hypertension. 1998;32(3):565-9.

14. Ziegelbauer K, Schaefer C, Steinmetz H, Sitzer M, Lorenz MW. Clinical usefulness of carotid ultrasound to improve stroke risk assessment: ten-year results from the Carotid Atherosclerosis Progression Study (CAPS). Eur J Prev Cardiol. 2013;20(5): 837-43.

15. Wilson PW, Zech LA, Gregg RE, Schaefer EJ, Hoeg JM, Sprecher DL, et al. Estimation of VLDL cholesterol in hyperlipidemia. Clin Chim Acta. 1985;151(3):285-91.

16. Payen T, Coron A, Lamuraglia M, Le Guillou-Buffello D, Gaud E, Arditi M, et al. Echo-power estimation from log-compressed video data in dynamic contrast-enhanced ultrasound imaging. Ultrasound Med Biol. 2013;39(10):1826-37.

17. Şehirli ÜS, Yalin A, Tulay CM, Cakmak YO, Gürdal E. The diameters of common carotid artery and its branches in newborns. Surg Radiol Anat. 2005;27(4):292-6.

18. Tokita A, Ishigaki Y, Okimoto H, Hasegawa H, Koiwa Y, Kato $\mathrm{M}$, et al. Carotid arterial elasticity is a sensitive atherosclerosis value reflecting visceral fat accumulation in obese subjects. Atherosclerosis. 2009;206(1):168-72.

19. Del Corso L, Moruzzo D, Conte B, Agelli M, Romanelli AM, Pastine F, et al. Tortuosity, kinking, and coiling of the carotid artery: expression of atherosclerosis or aging? Angiology. 1998;49(5):361-71.

20. Jartti L, Rönnemaa T, Kaprio J, Järvisalo MJ, Toikka JO, Marniemi J, et al. Population-based twin study of the effects of migration from Finland to Sweden on endothelial function and intima-media thickness. Arterioscler Thromb Vasc Biol. 2002;33(5):832-7.

21. Krejza J, Arkuszewski M, Kasner SE, Weigele J, Ustymowicz A, Hurst RW, et al. Carotid artery diameter in men and women and the relation to body and neck size. Stroke. 2006;37(4):1103-5.

22. Zhao J, Cheema FA, Bremner JD, Goldberg J, Su S, Snieder H, et al. Heritability of carotid intima-media thickness: a twin study. Atherosclerosis. 2008;197(2):814-20.

23. Liang L-R, Wong ND, Shi P, Zhao L-C, Wu L-X, Xie G-Q, et al. Cross-sectional and longitudinal association of cigarette smoking with carotid atherosclerosis in Chinese adults. Prev Med. 2009;49(1):62-7.

24. Kweon SS, Lee YH, Shin MH, Choi JS, Rhee JA, Choi SW, et al. Effects of cumulative smoking exposure and duration of smoking cessation on carotid artery structure. Circ J. 2012;76(8):2041-7.

25. Le Bret E, Pineau E, Folliguet T, Garabédian EN, Brunelle F, Vouhé $\mathrm{P}$, et al. Congenital kinking of the internal carotid artery in twin brothers. Circulation. 2000;102(22):E173-4.

26. Zhao S, Wang Y, Mu Y, Yu B, Ye P, Yan X, et al. Prevalence of dyslipidaemia in patients treated with lipid-lowering agents in China: results of the DYS lipidemia International Study (DYSIS). Atherosclerosis. 2014;235(2):463-9.

27. Salonen R, Nyyssönen K, Porkkala E, Rummukainen J, Belder R, Park JS, et al. Kuopio Atherosclerosis Prevention Study (KAPS). A population-based primary preventive trial of the effect of LDL lowering on atherosclerotic progression in carotid and femoral arteries. Circulation. 1995;92(7):1758-64.

28. Okamura T, Kokubo Y, Watanabe M, Higashiyama A, Miyamoto Y, Yoshimasa Y, et al. Low-density lipoprotein cholesterol and non-high-density lipoprotein cholesterol and the incidence of 
cardiovascular disease in an urban Japanese cohort study: the Suita study. Atherosclerosis. 2009;203(2):587-92.

29. Ikkruthi S, Rajappa M, Nandeesha H, Satheesh S, Sundar I, Ananthanarayanan $\mathrm{PH}$, et al. Hyperhomocysteinemia and hyperlipoproteinemia (a) in obese South Indian men: an indication for increased cardiovascular risk. Acta Physiol Hung. 2014;101(1):13-20.
30. van Popele NM, Grobbee DE, Bots ML, Asmar R, Topouchian J, Reneman RS, et al. Association between arterial stiffness and atherosclerosis: the Rotterdam Study. Stroke. 2001;32(2):454-60.

31. Sawabe M, Takahashi R, Matsushita S, Ozawa T, Arai T, Hamamatsu A, et al. Aortic pulse wave velocity and the degree of atherosclerosis in the elderly: a pathological study based on 304 autopsy cases. Atherosclerosis. 2005;179(2):345-51. 